\title{
Effects of Water Immersion in Different Water Depths on Respiratory Function and Respiratory Muscle Strength among Elderly People: An Observational Study
}

\author{
Yoshihiro Yamashina ${ }^{1}$, Tomoko Hirayama1, Hiroki Aoyama1, Hirofumi Hori², Emiko Morita1, \\ Nami Sakagami' ${ }^{1}$, Wataru Nanikawa1, Shigeru Terada1, Masahiro Goto', Kazuyuki Tabira ${ }^{3}$ \\ ${ }^{1}$ Department of Physical Therapy, Aino University, Osaka, Japan \\ ${ }^{2}$ Department of Physical Therapy, Biwako Professional University of Rehabilitation, Shiga, Japan \\ ${ }^{3}$ Department of Physical Therapy, Kio University, Nara, Japan \\ Email: *y-yamashina@pt-u.aino.ac.jp
}

How to cite this paper: Yamashin, Y., Hirayama, T., Aoyama, H., Hori, H., Morita, E., Sakagami, N., Nanikawa, W., Terada, S., Goto, M. and Tabira, K. (2021) Effects of Water Immersion in Different Water Depths on Respiratory Function and Respiratory Muscle Strength among Elderly People: An Observational Study. Advances in Aging Research, 10, 71-77.

https://doi.org/10.4236/aar.2021.104004

Received: April 27, 2021

Accepted: July 2, 2021

Published: July 5, 2021

Copyright $\odot 2021$ by author(s) and Scientific Research Publishing Inc. This work is licensed under the Creative Commons Attribution International License (CC BY 4.0).

http://creativecommons.org/licenses/by/4.0/

\begin{abstract}
The aim was to investigate the effect of three water levels (umbilical, $4^{\text {th }}$ rib, and clavicular) on the respiratory function and respiratory muscle strength among elderly. Spirometry and respiratory strength were measured on land as baseline data. Next, water depth conditions were determined randomly, and spirometry and respiratory muscle strength were measured at each water level. The Vital Capacity and Expiratory reserve volume in the clavicular level were significantly lower than those in the land and umbilical trials. No significant difference was observed in other respiratory functions. Chest circumference and respiratory muscle strength were not significantly different among all conditions.
\end{abstract}

\section{Keywords}

Respiratory Functions, Respiratory Muscle Strength, Water Immersion, Elderly People

\section{Introduction}

Aquatic exercise has recently been introduced as a part of health enhancement plans in rehabilitation and sports clubs [1] [2]. Aquatic exercise can reduce the self-weight load, thus allowing persons with obesity, joint diseases, or lumbago to perform exercise safely [3] [4]. In addition, water viscosity and pressure can be used as exercise loads to enhance extremity muscle strength [5] [6]. 
Breathing underwater requires great effort mainly for the following two reasons: first, the blood volume shifts into the chest cavity due to increased venous return from the lower extremities; and second, the chest wall and diaphragm inflexibility shifts toward the cranial side due to the hydrostatic pressure, resulting in restricted pulmonary compliance [7] [8]. Regarding the effects of water depth on respiratory function, the pulmonary vital capacity (VC), forced expiratory volume during the first second $\left(\mathrm{FEV}_{1.0}\right)$, and functional residual capacity (FRC) decrease during water immersion at the clavicular or cervical level [9] [10]. Furthermore, de Andrade et al. reported that decreased maximum inspiratory muscle strength during water immersion was greater when the water level was at the clavicle than at the xiphoid process due to the higher water pressure at the clavicular level (CL) [11]. We also suggested that forced respiration during upright water immersion up to the CL resulted in greater inspiratory muscle fatigue than at shallower depths in healthy young men [12].

As mentioned above, underwater environment exercises have been incorporated in various rehabilitation programs and are widely adopted in respiratory, heart, and other diseases. However, although several studies have investigated the effect of different water depths on respiratory function in young people, studies investigating this effect in elderly people are limited and with unclear findings.

Therefore, this study aimed to investigate the effect of three water levels (umbilical, $4^{\text {th }}$ rib, and clavicular) on the respiratory function and respiratory muscle strength among elderly people.

\section{Patients, Material and Methods}

\subsection{Participants}

A total of 14 elderly people were included in the study. Participants with history of respiratory or cardiovascular disease, hypertension (resting systolic blood pressure [BP] of $\geq 140 \mathrm{mmHg}$ and/or diastolic $\mathrm{BP}$ of $\geq 90 \mathrm{mmHg}$ ), diabetes, or obesity (body mass index [BMI] of $\geq 30 \mathrm{~kg} / \mathrm{m}^{2}$ ), or smoking habit were excluded. In addition, those with kyphosis were excluded to eliminate the influence of spinal deformity. Eligible participants who met the inclusion criteria were included in the study after familiarizing themselves with the experimental protocol, such as the measurement method using spirometry described below. Of the 14 registrants, two had a history of smoking and two had kyphosis and hence, they were excluded from the study. Therefore, statistical analyses were carried out using data from 10 participants. The participant characteristics are summarized in Table 1.

This study was approved by Aino University Research Ethics Committee and conformed to the standard set by the Declaration of Helsinki. Written informed consent was obtained from all participants prior to the experiment.

\subsection{Material and Methods}

An underwater treadmill (HOKKODENKI treadmill KRT-2500P) was used as 
Table 1. Participants characteristics.

\begin{tabular}{cc}
\hline Number & 10 \\
Age (years) & $67.3 \pm 2.2$ \\
Height $(\mathrm{m})$ & $1.65 \pm 0.06$ \\
Weight $(\mathrm{kg})$ & $65.5 \pm 7.6$ \\
BMI $\left(\mathrm{kg} / \mathrm{m}^{2}\right)$ & $23.8 \pm 2.1$ \\
Data are means \pm SD & \\
\hline
\end{tabular}

the water tank, and the water level was set to three settings: umbilical level (UL), $4^{\text {th }}$ rib level (RL), and CL trials. The water temperature was $32^{\circ} \mathrm{C} \pm 1^{\circ} \mathrm{C}$. As measurement items, VC, inspiratory capacity (IC), inspiratory reserve volume (IRV), and expiratory reserve volume (ERV) were measured using a spirometer (AS-507, Minato., Osaka, Japan) as respiratory function indices. Maximum inspiratory (PImax) and expiratory (PEmax) pressures in the oral cavity were also evaluated using a spirometer with the measuring unit of the respiratory muscle strength (Chest spirometer HI801) and considered as surrogate indices of inspiratory and expiratory muscle strength, respectively. The chest circumference (CC) was measured at the xiphoid process level.

Measurements were performed according to the protocol below. At the laboratory, participants' weight and height were measured before the first experiment. BMI $\left(\mathrm{kg} / \mathrm{m}^{2}\right)$ was calculated as body weight $(\mathrm{kg})$ divided by height $(\mathrm{m})$ squared. Then, participants practiced spirometry and respiratory muscle strength measurement methods, and all of them were trained. Then, 30 minutes after completing the practice, the experiment was started. Spirometry, respiratory strength, and chest circumference were measured 10 minutes after sitting on land and recorded as baseline data. Next, water depth conditions were determined randomly, and spirometry and respiratory muscle strength were measured $10 \mathrm{mi}-$ nutes after assuming a sitting position at each water level.

\subsection{Statistical Analysis}

All statistical analyses were performed using the StatView statistical software package (Ver5.0, SAS, Cary, NC, USA). Data were expressed as mean \pm standard deviation (SD) unless otherwise indicated. One-way analysis of variance with repeated measurements and subsequent multiple pairwise comparisons (Scheffe method) was performed to evaluate the effect of upright water immersion at different water depths on the respiratory function and respiratory muscle strength. Statistical significance was defined by a $\mathrm{P}$ value of $<0.05$.

\section{Results}

\section{Effects of Water Immersion at Different Water Depths on the Respiratory Function and Respiratory Muscle Strength}

Table 2 shows the respiratory function and respiratory muscle strength parameters 
Table 2. The effect of water immersion on the respiratory function and respiratory muscle strength.

\begin{tabular}{ccccc}
\hline & Land & Umbilical level & $4^{\text {th }}$-rib level & Clavicular level \\
\hline VC (L) & $3.6 \pm 0.3$ & $3.7 \pm 0.4$ & $3.5 \pm 0.5$ & $3.3 \pm 0.3^{* *}$ \\
IC (L) & $2.1 \pm 0.4$ & $2.1 \pm 0.5$ & $2.2 \pm 0.4$ & $2.5 \pm 0.3$ \\
ERV (L) & $1.5 \pm 0.3$ & $1.6 \pm 0.2$ & $1.4 \pm 0.3$ & $0.7 \pm 0.5^{* \#}$ \\
CC (cm) & $78.1 \pm 5.4$ & $77.7 \pm 5.2$ & $77.0 \pm 6.7$ & $77.3 \pm 3.1$ \\
PEmax ( $\left.\mathrm{cmH}_{2} \mathrm{O}\right)$ & $81.4 \pm 11.6$ & $80.7 \pm 11.5$ & $82.9 \pm 18.1$ & $81.5 \pm 12.7$ \\
PImax $\left(\mathrm{cmH}_{2} \mathrm{O}\right)$ & $90.2 \pm 14.6$ & $88.9 \pm 13.6$ & $87.8 \pm 16.3$ & $90.4 \pm 15.1$ \\
\hline
\end{tabular}

Values are means \pm SD. ${ }^{\star} \mathrm{p}<0.05$ vs. land, ${ }^{*} \mathrm{p}<0.05$ vs. umbilical level. Abbreviations: VC, vital capacity; IC, inspiratory capacity; ERV, expiratory reserve volume; CC, chest circumference; PEmax, maximal expiratory pressure; PImax, maximal inspiratory pressure.

on land and during water immersion. The VC and ERV in the CL were significantly lower than those in the land and UL trials. No significant difference was observed in other respiratory functions. Chest circumference and respiratory muscle strength were not significantly different among all conditions.

\section{Discussion}

This study demonstrated that VC and ERV in the CL were significantly lower than those in the land and UL trials. Agostoni et al. stated that respiratory functions, such as VC and forced expiratory volume in $1 \mathrm{~s}$ of water immersion, were reduced in proportion to the water depth and that the reduction become remarkable when the depth reached the CL [9]. Dahlback and Buono et al. also reported that VC was significantly decreased by water immersion at the CL [13] [14]. Our results are consistent with those of previous studies. Kurabayashi et al. reported that CC was reduced by $0.5 \mathrm{~cm}$ underwater at the $\mathrm{CL}$ depth than that before submergence [15]; however, our results did not show a significant difference regardless of water depth. Chest compliance has been reportedly reduced in elderly people [16]. Therefore, they are less likely susceptible to CC changes due to hydraulic pressure on the chest wall. The abdominal and chest walls are speculated to be compressed by hydrostatic pressure during water immersion, resulting in a cranial shift of the diaphragm, which decreased VC. This cranial shift also minimizes the alveolar size at the end-expiratory phase, which may decrease ERV.

In this study, neither PImax nor PEmax was affected just by water immersion at any depth. In terms of PImax, our results were consistent with that of Schoenhofer et al.'s, which showed that PImax tended to decrease from the baseline after water immersion at the CL depth. PImax is generally recommended to be measured at the maximum expiratory phase based on the length-tension relationship theory [17]. Briefly, the maximum expiratory phase is determined by the balance between expiratory muscle contraction and opposing elastic dilatation pressure of the lungs and thorax. At this phase, the inspiratory muscles and 
diaphragm are extended to the maximum and demonstrate maximal tensile strength to generate maximal inspiratory pressure with support from the elastic dilatation pressure in the lungs and thorax. Water immersion could promote easy retention in the maximum expiratory phase; this may be the reason why PImax was not impaired by water immersion in this study. In terms of PEmax, our results support Andrade et al.'s findings that regardless of the water depth, PEmax during water immersion was not different at the iliac crest, xiphoid process, or CL [11]. Primarily, the decreased VC that is accompanied by submergence may be unfavorable to measure PEmax from the above length-tension relationship perspective. However, hydrostatic pressure against the entire thorax at the CL depth is speculated to have assisted expiration, resulting in the minimum influence on PEmax.

There are some limitations in the present study. The results of the present study with the small number of subjects suggest that VC and ERV decreased when water immersion was at the CL. We will increase the number of subjects more from now on, and have to consider whether it'll be a similar result. In addition, water resistance changes depending on the movement speed, moving with low resistance may be possible by changing the speed even if the movement is the same. Therefore, when performing respiratory exercises underwater, the exercise load may be reduced if the inspiratory speed is slow. Further detailed exercise methods that affect the respiratory function should be investigated in future studies.

\section{Conclusion}

In conclusion, we demonstrated that VC and ERV decreased when water immersion was at the CL in the elderly, but did not affect the respiratory muscle strength. Further studies are needed to develop an exercise regimen that can be utilized in the field of health promotion for elderly that makes the most of the characteristics of submersion and aquatic exercise.

\section{Acknowledgements}

We are grateful to members of Aino University for their important contributions to this experiment. This work was supported by Japan Society for the Promotion of Science (JSPS), KAKENHI, Grants-in-Aid for Young Scientists.

\section{Conflicts of Interest}

The authors declare no conflicts of interest regarding the publication of this paper.

\section{References}

[1] Cider, A., Sunnerhagen, K.S., Schaufelberger, M. and Andersson, B. (2005) Cardiorespiratory Effects of Warm Water Immersion in Elderly Patients with Chronic Heart Failure. Clinical Physiology and Functional Imaging, 25, 313-317. https://doi.org/10.1111/j.1475-097X.2005.00633.X 
[2] Wilcock, I.M., Cronin, J.B. and Hing, W.A. (2006) Physiological Response to Water Immersion: A Method for Sport Recovery? Sports Medicine, 36, 747-765. https://doi.org/10.2165/00007256-200636090-00003

[3] Chi, D., Back, Y., Park, G., Ju, S. and Jang, H. (2011) The Effect of Aquatic Exercise on Peak Torque and Stability of Knee Joints of Elderly Women. The Journal of Physical Therapy Science, 23, 871-873. https://doi.org/10.1589/jpts.23.871

[4] Irandoust, K. and Taheri, M. (2015) The Effects of Aquatic Exercise on Body Composition and Nonspecific Low Back Pain in Elderly Males. The Journal of Physical Therapy Science, 27, 433-435. https://doi.org/10.1589/jpts.27.433

[5] Poyhonen, T., Sipila, S., Keskinen, K.L., Hautala, A., Savolainen, J. and Malkia, E. (2002) Effects of Aquatic Resistance Training on Neuromuscular Performance in Healthy Women. Medicine \& Science in Sports \& Exercise, 34, 2103-2109. https://doi.org/10.1097/00005768-200212000-00036

[6] Wang, T.J., Belza, B., Thompson, F.E., Whitney, J.D. and Bennett, K. (2007) Effects of Aquatic Exercise on Flexibility, Strength and Aerobic Fitness in Adults with Osteoarthritis of the Hip or Knee. Journal of Advanced Nursing, 57, 141-152. https://doi.org/10.1111/j.1365-2648.2006.04102.x

[7] Craig, A.B. and Ware, D.E. (1967) Effect of Immersion in Water on Vital Capacity and Residual Volume of Lungs. Journal of Applied Physiology, 23, 423-425. https://doi.org/10.1152/jappl.1967.23.4.423

[8] Delafargue, A., Schmid, B., Similowski, T. and Regnard, J. (2021) Broad Individual Immersion-Scattering of Respiratory Compliance Likely Substantiates Dissimilar Breathing Mechanics. Scientific Reports, 11, Article No. 9434. https://doi.org/10.1038/s41598-021-88925-x

[9] Agostoni, E., Gurtner, G., Torri, G. and Rahn, H. (1966) Respiratory Mechanics during Submersion and Negative-Pressure Breathing. Journal of Applied Physiology, 21, 251-258. https://doi.org/10.1152/jappl.1966.21.1.251

[10] Prefaut, C., Lupih, E. and Anthonisen, N.R. (1976) Human Lung-Mechanics during Water Immersion. Journal of Applied Physiology, 40, 320-323.

https://doi.org/10.1152/jappl.1976.40.3.320

[11] de Andrade, A.D., Junior, J.C., Lins de Barros Melo, T.L., Rattes Lima, C.S., Brandao, D.C. and de Melo Barcelar, J. (2014) Influence of Different Levels of Immersion in Water on the Pulmonary Function and Respiratory Muscle Pressure in Healthy Individuals: Observational Study. Physiotherapy Research International, 19, 140-146. https://doi.org/10.1002/pri.1574

[12] Yamashina, Y., Yokoyama, H., Naghavi, N., Hirasawa, Y., Takeda, R., Ota, A., Imai, D., Miyagawa, T. and Okazaki, K. (2016) Forced Respiration during the Deeper Water Immersion Causes the Greater Inspiratory Muscle Fatigue in Healthy Young Men. The Journal of Physical Therapy Science, 28, 412-418.

https://doi.org/10.1589/jpts.28.412

[13] Dahlback, G.O., Jonsson, E. and Liner, M.H. (1978) Influence of Hydrostatic Compression of the Chest and Intrathoracic Blood Pooling on Static Lung Mechanics during Head-Out Immersion. Undersea Biomedical Research, 5, 71-85.

[14] Buono, M.J. (1983) Effect of Central Vascular Engorgement and Immersion on Various Lung-Volumes. Journal of Applied Physiology, 54, 1094-1096. https://doi.org/10.1152/jappl.1983.54.4.1094

[15] Kurabayashi, H., Tamura, K., Kubota, K. and Tamura, J. (2001) Analysis of the Circumferences of Chest, Abdomen, Thigh and Calf during Head-Out Water Immersion. The Journal of the Japanese Society of Balneology, Climatology and Physical Medi- 
cine, 64, 199-202.

[16] Sharma, G. and Goodwin, J. (2006) Effect of Aging on Respiratory System Physiology and Immunology. Clinical Interventions in Aging, 1, 253-260.

https://doi.org/10.2147/ciia.2006.1.3.253

[17] Agostoni, E. and Mead, J. (1973) Statics of the Respiratory System. American Physiological Society, Washington DC, 387-410. 